\title{
Abnormal expression of FOSB correlates with tumor progression and poor survival in patients with gastric cancer
}

\author{
CHONG TANG $^{1^{*}}$, YASU JIANG $^{1 *}$, WEIWEI SHAO ${ }^{3 *}$, WEN SHI $^{1}$, XUESONG GAO $^{1}$, \\ WEIYAN QIN $^{1}$, TIAN JIANG ${ }^{1}$, FEIRAN WANG ${ }^{2}$ and SHICHUN FENG ${ }^{1}$ \\ ${ }^{1}$ Department of General Surgery, The Second Affiliated Hospital of Nantong University, Nantong University; \\ ${ }^{2}$ Department of General Surgery, The Affiliated Hospital of Nantong University, Nantong University, \\ Nantong, Jiangsu 226001; ${ }^{3}$ Department of General Surgery, The Fourth Affiliated Hospital of \\ Nantong University, Nantong University, Yancheng, Jiangsu 224000, P.R. China
}

Received May 4, 2016; Accepted July 20, 2016

DOI: 10.3892/ijo.2016.3661

\begin{abstract}
FOSB protein is encoded by the FOSB gene in humans, which shares structural similarities with the prototype of the Fos family. FOSB plays a role by AP-1 complex which is composed of heterodimers of Jun and Fos members. Our experiment aimed to evaluate the effect of FOSB in gastric cancer (GC) patients and then probe its significance in prognosis. We detected the expression of FOSB in GC and adjacent non-cancerous tissues by western blot analysis and real-time quantitative PCR (qRT-PCR). Moreover, we analyzed FOSB expression in patients who underwent resection procedures using immunohistochemistry. The relationship between the expression of FOSB, the clinicopathological characteristics and the patients survival were also investigated. Furthermore, in vitro, we evaluated the effects of FOSB gene on gastric cancer cell viability, proliferation and migration by MTT, clone formation and Transwell assays. Finally, the Kaplan-Meier method and log-rank test were used to compare the overall survival between high FOSB expression group and low FOSB expression group. Immunohistochemical staining data showed that FOSB expression was significantly decreased in gastric cancer cases. In addition, we confirmed FOSB downregulation in both mRNA and protein levels in GC tissues compared with matched adjacent non-cancerous tissues. Downregulated expression of FOSB was correlated with poor differentiation,
\end{abstract}

Correspondence to: Dr Feiran Wang, Department of General Surgery, The Affiliated Hospital of Nantong University, Nantong University, Nantong, Jiangsu 226001, P.R. China

E-mail: wangfr0830@sina.com

Dr Shichun Feng, Department of General Surgery, The Second Affiliated Hospital of Nantong University, 6 Haierxiang Road, Nantong, Jiangsu 226001, P.R. China

E-mail: fengshichungs@sina.com

*Contributed equally

Key words: gastric cancer, FOSB, prognosis lymph node metastasis and advanced TNM stage. Moreover, we found that low FOSB expression exhibited a significant correlation with poor prognosis for GC patients by KaplanMeier survival analysis. Overexpression of FOSB significantly suppressed cell proliferation, clone formation and migration in GC cell lines. In contrast, silencing of FOSB expression in GC cells promoted proliferation, clone formation and migration. Our results showed that FOSB plays a crucial role in the suppression of GC, and that it may be a useful biomarker in diagnosis and prognosis for GC patients.

\section{Introduction}

According to WHO, gastric cancer (GC) is the fourth most common cancer and the second most common cause of cancerrelated death worldwide (1). In China, gastric cancers are considered to be the second most frequently diagnosed cause of cancer death (2). Recently, owing to the global environment worsening and change in people's habits, incidence of gastric cancer has increased year by year. Despite some advances in diagnosis, staging and treatment of GC, especially surgery, chemotherapy and radiotherapy, the long-term survival rate remains quite low because of local invasion and distant metastasis. The incidence, development, infiltration and metastasis of GC are a multistep and multifactor process. It may be regulated by many genes and involves a variety of gene activation, regulated disorder or inactivation (3). Recently, many characteristic biological markers are being studied, which may be very helpful to clinicians to predict metastatic progression and prognosis of GC patients.

FBJ murine osteosarcoma viral oncogene homolog B, also known as FOSB (in humans) or FosB (in other species), is a protein that is encoded by the FOSB gene that is localized on chromosome 19q13 and is composed of four exons in humans $(4,5)$. FosB is an acidic protein of 338 amino acids which shares structural similarities with the prototype of the Fos family, c-Fos, namely, a prolin-rich basic DNA-binding region, a leucine zipper required for dimer formation, and a C-terminal transactivation domain (6). FOSB gene encodes leucine zipper proteins that dimerize with proteins of the JUN family, thereby forming the transcription factor complex 
AP-1. AP-1 regulates gene expression in response to a variety of stimuli, including cytokines, growth factors, stress and bacterial and viral infections (7). It was shown in various experimental systems that stronger proliferation, malignant transformation and enhanced aggressiveness are accompanied by a change in AP-1 complex composition (8-10). Similarly, the inhibition of proliferation and induction of differentiation processes leads to a shift of expression of the individual AP-1 proteins (11-13). In the past several years, some studies identified that downregulated expression of FOSB is frequent in breast carcinomas (14) and pancreatic cancer (15). Up to now, there has not been research reporting the role of FOSB in GC. Thus, the purpose of the present study was to determine FOSB expression in human GC patient specimens and then to evaluate the clinicopathological implications of FOSB expression in GC. Furthermore, we investigated the potential influence of FOSB in the development of GC.

\section{Materials and methods}

Tissue collection. We retrospectively analyzed clinicopathological data from 116 gastric cancer patients who underwent surgical resection at the Second Affiliated Hospital of Nantong University from January 2008 to December 2010, and obtained fresh gastric cancer and surrounding non-cancerous tissue samples randomly from $40 \mathrm{GC}$ patients. After surgical resection, fresh samples were frozen in liquid nitrogen immediately and divided into two parts, and one was maintained at $-80^{\circ} \mathrm{C}$ until use for real-time PCR, the other used for western blot analysis. Paraffin-embedded samples were obtained from 116 GC patients for immunohistochemistry. The clinical information related to the $116 \mathrm{GC}$ patients, including gender, age, tumor size, TNM stage and lymph node metastasis was also collected. Staging and grading were referred to the classification of the International Union against Cancer (UICC) criteria. None of the patients were administered preoperative radiotherapy or chemotherapy. All human tissues were collected using protocols approved by the Ethics Committee of The Second Affiliated Hospital of Nantong University.

Western blot analysis. Paired cancer and their paracancerous tissue specimens were used for western blot analysis. We used lysis buffer which contain protease inhibitors (Promega, Madison, WI, USA) to extract total protein. Then, equal amounts $(30 \mu \mathrm{g})$ of protein separated by $10 \%$ sodium dodecyl sulfate polyacrylamide gel electrophoresis (SDS-PAGE). After that, they were transferred to a polyvinylidene fluoride (PVDF) membrane, and we used 5\% non-fat milk in TBST (Tris-buffered saline containing $0.1 \%$ Tween-20) to block non-specific binding for $2 \mathrm{~h}$. After the incubation with the primary antibodies overnight at $4^{\circ} \mathrm{C}$ [a rabbit polyclonal anti-human FOSB antibody (1:100, sc-48; Santa Cruz Biotechnology, Inc., Santa Cruz, CA, USA) or a rabbit anti- $\beta$-actin as internal reference, at 1:2,000 dilution (Sigma-Aldrich, St. Louis, MO, USA)], membranes were washed three times in TBST for $5 \mathrm{~min}$ and then incubated with horseradish peroxidase-conjugated goat anti-rabbit secondary antibody (1:1,000 dilution; Sigma-Aldrich) for $2 \mathrm{~h}$ at room temperature. Finally signals were scanned with an Odyssey infrared imaging system (LI-COR Biosciences,
Lincoln, NE, USA), and analyzed with PDQuest 7.2.0 software (Bio-Rad Laboratories, Hercules, CA, USA).

Quantitative real-time PCR. The mRNA expression of FOSB was analyzed by the quantitative real-time PCR. Total RNAs were isolated from tumor tissue samples (100-200 mg) adjacent to the region which was used for protein extraction. Quantitative real-time PCR was performed using HotStart-IT SYBR-Green qPCR Master Mix (2X; USB Corp., Cleveland, $\mathrm{OH}, \mathrm{USA})$. On the basis of the HotStart-IT protocol, $25 \mu \mathrm{l}$ reactions were run with $2 \mu \mathrm{l}$ of cDNA. In addition, RT-PCR experiments were performed in a LightCycler 480 system (Roche Applied Science). PCR procedures: first, hot start at $95^{\circ} \mathrm{C}$ for $10 \mathrm{~min}$; 40 cycles of amplification/quantification at $95^{\circ} \mathrm{C}$ for $10 \mathrm{sec}$, then $60^{\circ} \mathrm{C}$ for $30 \mathrm{sec}$, and $72^{\circ} \mathrm{C}$ for $30 \mathrm{sec}$ when fluorescence was measured. Melting curve analysis was performed using continuous fluorescence acquisition from 65 to $97^{\circ} \mathrm{C}$. These cycling parameters generated single amplicons for both primer sets used according to the presence of a single melt peak. The sequences of the primers for FOSB were: FOSB forward, 5'-TGACAGTGTTATCCCAAGACCC-3' and FOSB reverse, 5'-CCAGCAGGACGGCATCA-3'. The $\beta$-actin was selected as the internal reference. All quantitative realtime PCRs were repeated three times for each gene and each sample was done in triplicate.

Immunohistochemistry. The tissue sections were deparaffinized with dimethylbenzene and rehydrated through 100, 95, 90,80 and $70 \%$ ethanol. After three washes in phosphate-buffered saline (PBS), the slides were boiled in antigen retrieval buffer containing $0.01 \mathrm{M}$ sodium citrate-hydrochloric acid for $10 \mathrm{~min}$ in a microwave oven. After rinsing with PBS, the tissue sections were incubated with a rabbit polyclonal antihuman FOSB antibody (1:100; Santa Cruz Biotechnology) and the slides were then rinsed in $3 \%$ hydrogen peroxide to block endogenous peroxidase. The sections were incubated with a donkey anti-rabbit second antibody conjugated horseradish peroxidase (1:5,000; Abcam, Cambridge, UK) at $4^{\circ} \mathrm{C}$ overnight and then incubated with horseradish peroxidase (HRP) (at room temperature for $30 \mathrm{~min}$. After washing in PBS, the visualization signal was developed with 3,3'-diaminobenzidine (DAB) solution, and all of the slides were counterstained with hematoxylin.

The total FOSB immunohistochemistry staining score was calculated as the sum of the percentage of positively stained cells and the staining intensity. Briefly, the percentage of positive staining was scored as 0 (0-9\%), 1 (10-25\%), 2 (26-50\%) or $3(51-100 \%)$, and the intensity was scored as 0 (no staining), 1 (weak staining), 2 (moderate staining) or 3 (strong staining). The expression level of FOSB was defined as follows: '-' (negative, score of 0 ), ' + ' (weak positive, score of 1-3), ' ++ ' (positive, score of 4-6) and ' +++ ' (strong positive, score of 7-9). We defined high FOSB expression as a total score of $>3$, and low FOSB expression as a total score of $<3$.

Cell culture, plasmid construction and transfection. The human GC cell lines, AGS, SGC7901, BGC823 and MKN45 and the human normal gastric epithelial mucosa cell line GES-1 were all obtained from the Cell Bank of the Committee on Type Culture Collection of the Chinese Academy of 
Sciences (Shanghai, China). All cell lines were grown in Dulbecco's modified Eagle's medium (DMEM) or RPMI-1640 medium (Gibco) with $10 \%$ fetal bovine serum (FBS; HyClone Laboratories, Inc., Logan, UT, USA) and were cultured in humidified incubator at $37^{\circ} \mathrm{C}$ with $5 \% \mathrm{CO}_{2}$.

For siRNA silencing of FOSB, two pairs of siRNA oligos for FOSB were designed and purchased from Shanghai GenePharma, Co., Ltd. (Shanghai, China). The targeting sequences were: FOSB siRNA1: 5'-AAGGGTGCGCCGGGA ACGAAATAAA-3'; FOSB siRNA2: 5'-GGAACGAAATAA ACTAGCAGCAGCT-3'; and FOSB siRNA3: 5'-GGCTTCT CTCTTTACACACAGTGAA-3'. Synthesized siRNAs were transfected into GC cells, respectively, using the Lipofectamine RNAiMAX transfection reagent (Invitrogen, Carlsbad, CA, USA) according to the manufacturer's instructions. In addition, siRNA3 could effectively reduce endogenous FOSB expression by western blotting assay. Therefore, siRNA3 was chosen for downstream experiment.

FOSB overexpression vector pcDNA 3.1(+)-FOSB was constructed using the PCR method. The PCR products were confirmed by direct DNA sequencing and cloned into the mammalian expression vector pcDNA 3.1(+). We obtained stably transfected clones by G418 selection (Promega). A stable transfectant of the pcDNA 3.1(+) vector was used as a control. For transfection, pcDNA 3.1(+)-FOSB expression plasmids were transfected into GC cells using Lipofectamine 2000 (Invitrogen) according to the manufacturer's instructions. The level of FOSB expression after transfection was analyzed by western blot analysis.

Cell viability assay. Cell viability was tested by using MTT assay. All cells were plated in 96 -well plates at $5 \times 10^{3}$ cells/ well in complete medium and cultured for $24 \mathrm{~h}$, and replaced with medium which contained $10 \%$ FBS, then $10 \mu \mathrm{l}$ MTT was added to each corresponding test well and the plates were further incubated for $4 \mathrm{~h}$. All samples were measured at $490 \mathrm{~nm}$ spectrophotometric absorbance. All experiments were performed in triplicate.

Colony formation assay. After transfection, cells $\left(5 \times 10^{4} /\right.$ well) were separately plated in a 24 -well plate. After $24 \mathrm{~h}$, the cells were collected and seeded (1,000-1,500/well) in a fresh 6 -well plate for 12 days. Surviving colonies ( $>50$ cells per colony) were counted after fixed with methanol/acetone (1:1) and stained with 5\% Gentian violet (ICM Pharma Pte. Ltd., Singapore, Singapore), after that rinsed three times with PBS to remove excess dye, photographed and counted. The experiment was carried out in triplicate wells three times.

Cell migration assay. According to the manufacturer's instructions, the cell migratory capacity was determined by Transwell chambers (BD Biosciences, San Jose, CA, USA). After transfection, cells were harvested at $24 \mathrm{~h}$ and then $3.0 \times 10^{5}$ transfected cells or untreated cells in serum-free medium were added to each upper insert. The chemotactic factor, DMEM medium (Gibco) which contained 10\% FBS (500 $\mu \mathrm{l})$ was added to the lower chamber. After incubation for $48 \mathrm{~h}$, nonmigrated cells on the upper surface were removed gently with a cotton swab and the migrated cells on the lower membrane surface were fixed in methanol. Cells were stained with $0.1 \%$

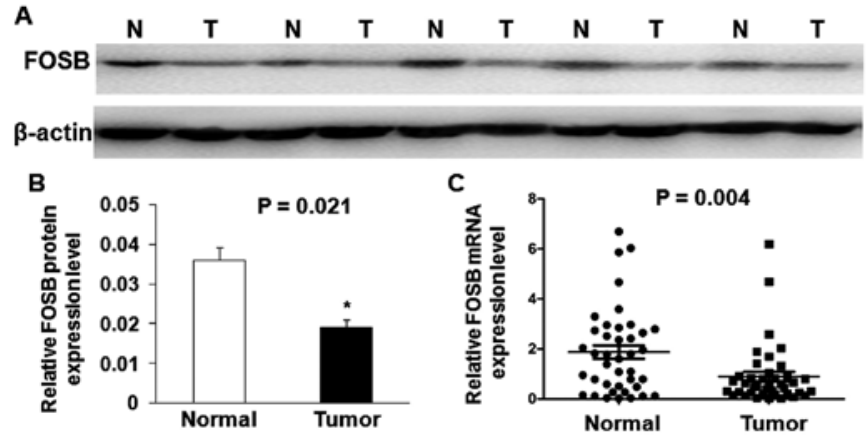

Figure 1. FOSB expression was remarkably downregulated in GC tissues. (A) Western blot analysis of FOSB proteins expressed in five paired representative GC tissues (T) and their matched adjacent non-cancerous tissues (N). $\beta$-actin was used as a loading control. (B) Relative average FOSB protein expression levels were significantly downregulated in 19 of $24(79.17 \%) \mathrm{GC}$ tissues compared with the corresponding adjacent non-cancerous tissues $\left({ }^{*} \mathrm{P}=0.021\right)$. (C) The average relative expression of mRNA level of FOSB in $\mathrm{GC}$ tissues compared to paired adjacent non-cancerous tissues $(\mathrm{P}=0.004)$.

crystal violet, photographed and counted. The experiment was performed in triplicate and repeated three times.

Statistical analysis. The SPSS 17.0 statistical software (SPSS, Inc., Chicago, IL, USA) was used for statistical analysis. The relationship between the FOSB expression level and the clinicopathological characteristics was subjected to the $\chi^{2}$ test. Survival curves were calculated by the Kaplan-Meier method and the differences were analyzed with the log-rank test. A multivariate analysis of several prognostic factors was carried out using the Cox proportional hazards regression model. The results were expressed as the mean \pm SD of at least three independent experiments, for all statistical analyses. P-value $<0.05$ was considered to be statistically significant.

\section{Results}

FOSB expression analyzed in gastric cancer tissue samples by western blot analysis and $q R T-P C R$. Twenty-four gastric cancer specimens as well as their adjacent non-cancerous tissues were selected from 40 gastric cancer patients randomly and used to evaluate FOSB protein expression by western blot analysis. The representative results of western blot analysis in five cases are shown in Fig. 1A. The results show that the expression of FOSB protein level was significantly downregulated in most of GC tissues $(79.17 \%, 19$ of 24) compared with adjacent non-cancerous tissues, and the average FOSB protein level in $24 \mathrm{GC}$ tissues was significantly lower than that in adjacent non-cancerous tissues $(\mathrm{P}=0.021$; Fig. 1B). We also performed qRT-PCR analysis in $40 \mathrm{GC}$ tissues and matched adjacent non-cancerous tissues. As shown in Fig. 1C, the average relative expression of FOSB mRNA level was significantly lower in GC tissues compared with adjacent noncancerous tissues $(\mathrm{P}=0.004)$.

To further investigate FOSB expression level, a total of 116 paraffin-embedded GC blocks were evaluated by IHC analysis. We found that FOSB was expressed at various levels in the GC tissues and the adjacent non-cancerous tissue samples (Fig. 2) Overall, 64 of $116(55.17 \%)$ cases showed low FOSB expression (FOSB - or FOSB +) in GC tissues, while the remaining 
A

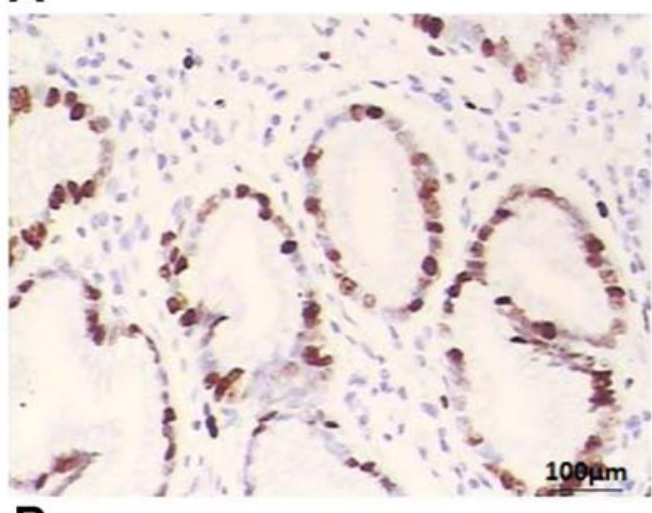

B

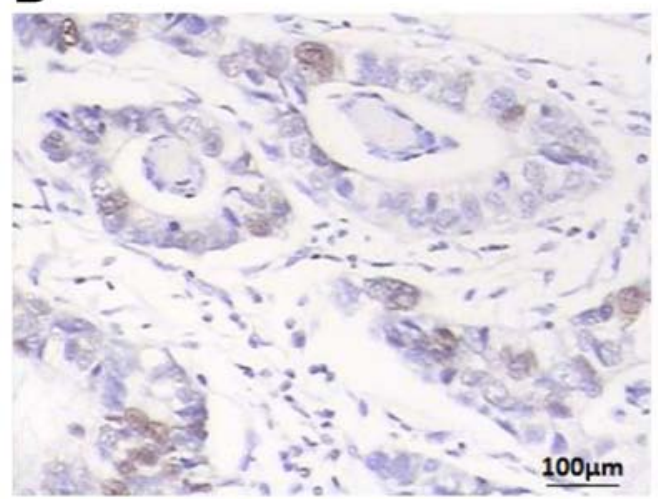

C

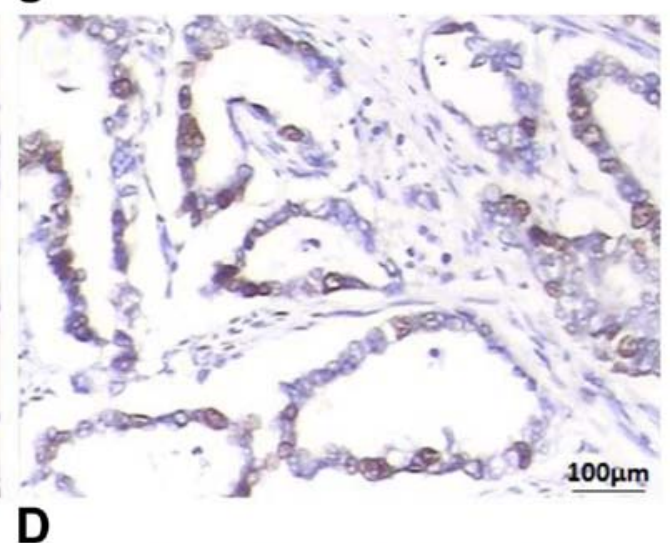

D

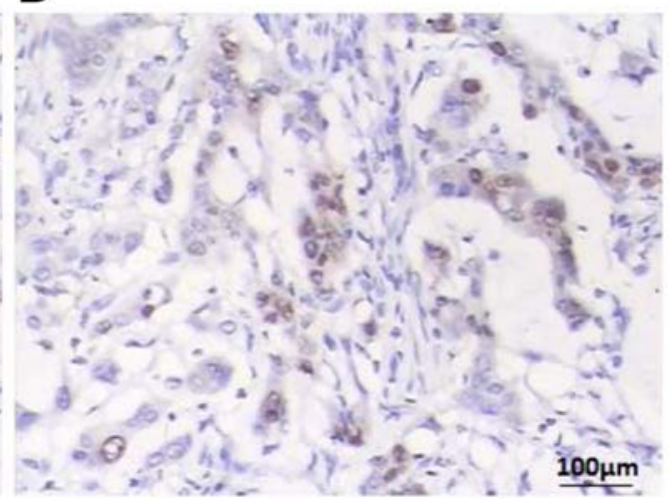

Figure 2. Immunohistochemical staining of FOSB in GC tissues and adjacent non-tumorous tissues. (A) Strong staining in adjacent non-tumor tissues, scored as FOSB (+++). (B) Moderate staining in well-differentiated GC tissues, scored as FOSB (++). (C) Weak staining in moderately differentiated GC tissues, scored as FOSB (+). (D) Negative staining in poorly differentiated GC tissues, scored as FOSB (-). Original magnification, x200.
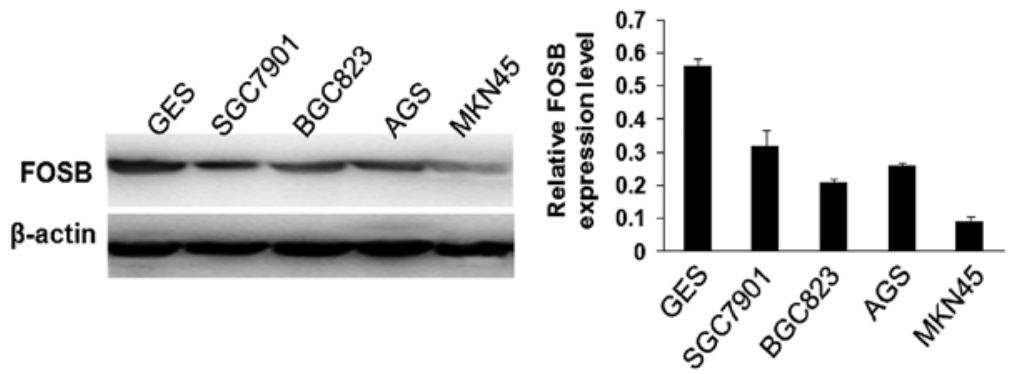

Figure 3. Western blotting showing the analysis of FOSB protein expression levels in the gastric cancer cell lines and the gastric epithelial mucosa cell line (GES-1).

$52(44.83 \%)$ cases displayed high SPOP expression (FOSB ++ or FOSB +++) (Table I). The adjacent non-cancerous tissues showed the strongest FOSB-positive staining (Fig. 2A).

Overexpression of FOSB inhibits gastric cancer cell viability, proliferation and migration in vitro. FOSB was significantly decreased in GC tissues and acted as a tumor suppressor gene. MKN45 cell line with the lowest expression of FOSB of the five tested GC cell lines (Fig. 3) was chosen to examine whether overexpression of FOSB affected cell viability, proliferation and migration in GC. The effects of FOSB on cell viability and proliferation were evaluated by MTT and colony formation assay, and the results showed that overexpression of FOSB suppressed the viability and the number of colonies of MKN45 cells (Fig. 4B and $\mathrm{C}$; $\mathrm{P}<0.05$ ). In addition, these results were further confirmed by assay, overexpression of FOSB could decrease the transformation phenotype of GC cells in vitro. Besides, we investigated the potential role of FOSB on cellular migration by Transwell assays. MKN45 cells were transfected with FOSB overexpressing plasmid or control plasmid and seeded in the chamber, and their migratory abilities were determined $24 \mathrm{~h}$ later. The results showed that the overexpression of FOSB inhibited the migratory capacity of MKN45 cells (Fig. 4C; $\mathrm{P}<0.05$ ).

Knockdown of FOSB expression promotes gastric cancer cell viability, proliferation and migration in vitro. To further study the effects of FOSB on the viability, proliferation and migra- 
Table I. Correlation between the FOSB expression and the clinicopathological characteristics in patients with gastric cancer.

\begin{tabular}{|c|c|c|c|c|}
\hline \multirow[b]{2}{*}{ Parameters } & \multirow[b]{2}{*}{$\begin{array}{c}\text { Total } \\
(\mathrm{n}=116)\end{array}$} & \multicolumn{2}{|c|}{$\begin{array}{c}\text { FOSB } \\
\text { expression }\end{array}$} & \multirow[b]{2}{*}{ P-value } \\
\hline & & $\begin{array}{l}\text { Low } \\
(n=64)\end{array}$ & $\begin{array}{l}\text { High } \\
(\mathrm{n}=52)\end{array}$ & \\
\hline Gender & & & & 0.507 \\
\hline Male & 72 & 38 & 34 & \\
\hline Female & 44 & 26 & 18 & \\
\hline Age (years) & & & & 0.978 \\
\hline$<60$ & 40 & 22 & 18 & \\
\hline$\geq 60$ & 76 & 42 & 34 & \\
\hline Tumor size (cm) & & & & 0.115 \\
\hline$<5$ & 54 & 34 & 20 & \\
\hline$\geq 5$ & 62 & 30 & 32 & \\
\hline Location & & & & 0.418 \\
\hline Cardia & 38 & 23 & 15 & \\
\hline Body/antrum & 78 & 41 & 37 & \\
\hline Histological grade & & & & $0.001^{\mathrm{a}}$ \\
\hline Well/moderate & 52 & 13 & 39 & \\
\hline Poor & 64 & 51 & 13 & \\
\hline Invasive depth & & & & 0.064 \\
\hline $\mathrm{T} 1 / 2$ & 45 & 20 & 25 & \\
\hline $\mathrm{T} 3 / 4$ & 71 & 44 & 27 & \\
\hline \multicolumn{5}{|l|}{ H. pylori infection } \\
\hline Negative & 30 & 16 & 14 & 0.814 \\
\hline Positive & 86 & 48 & 38 & \\
\hline Lymph node metastasis & & & & $0.001^{\mathrm{a}}$ \\
\hline Negative & 34 & 11 & 23 & \\
\hline Positive & 82 & 53 & 29 & \\
\hline TNM stage & & & & $0.021^{\mathrm{a}}$ \\
\hline $\mathrm{I} / \mathrm{II}$ & 69 & 32 & 37 & \\
\hline III/IV & 47 & 32 & 15 & \\
\hline Distant metastasis & & & & 0.243 \\
\hline Negative & 108 & 58 & 50 & \\
\hline Positive & 8 & 6 & 2 & \\
\hline
\end{tabular}

aP-value $<0.05$ was considered statistically significant.

tion of gastric cancer cells, SGC7901 cells that possessed the highest FOSB expression in the four GC cells (Fig. 3) were transfected with FOSB siRNA1, FOSB siRNA2 and FOSB siRNA3. Western blot analysis showed that siRNA3 reduced the level of endogenous FOSB expression more significantly than siRNA1 and siRNA2 (Fig. 5A). The effects of FOSB on cell viability and proliferation was assessed by MTT and colony formation assays, and the results indicated that downregulation of FOSB expression promoted the viability of SGC7901 cells and increased the number of colonies of
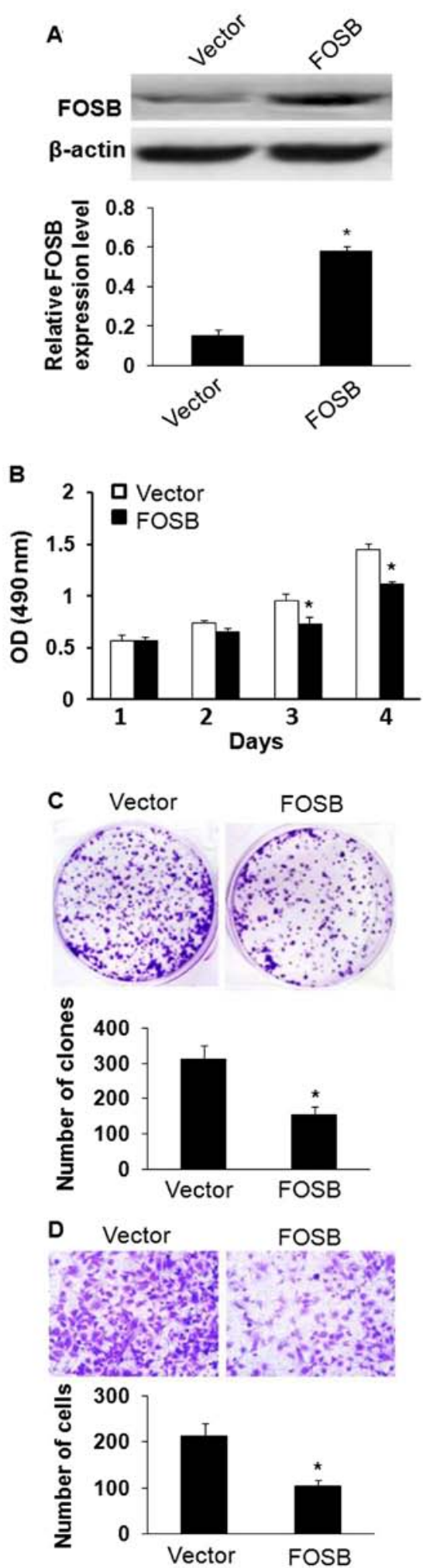

Figure 4. Overexpression of FOSB inhibited the viability, proliferation and migration of GC cells in vitro. (A) Western blot analysis of FOSB protein expression levels. The FOSB overexpression transfectant (FOSB) and the empty vector transfectant (vector) are shown. (B) Cell viability was evaluated with MTT assay using absorbance readings at $490 \mathrm{~nm}$. MKN45 cells were transfected with pcDNA3.1 (+)-FOSB or empty vector for 1, 2, 3 and 4 days, respectively. The values are indicated by the mean of three determinations. (C) Colony numbers of the MKN45/FOSB and control cells in plate colony formation assays. The data represent the means \pm SEM of three independent experiments. (D) Transwell assays demonstrated that overexpression of FOSB in MKN45 cells transfected with pcDNA3.1 (+)-FOSB plasmid suppressed cell migration compared with empty vector $\left({ }^{*} \mathrm{P}<0.05\right)$. Representative photos of stained cells are shown with the original magnification of $\mathrm{x} 100$. 
A
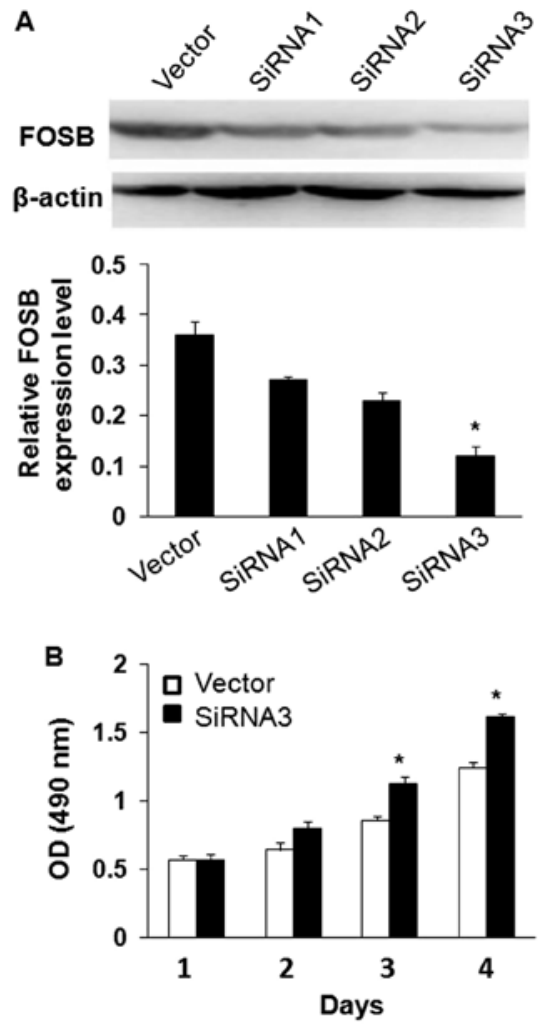

C
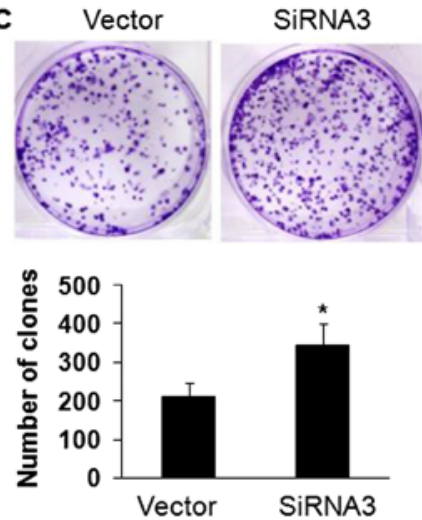

D

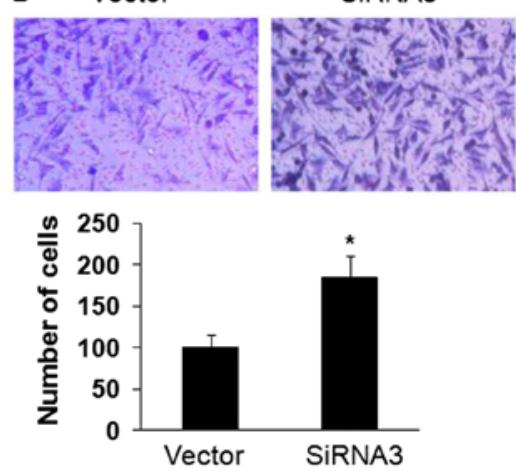

Figure 5. Knockdown of FOSB expression stimulated cell viability, proliferation and migration in vitro by using siRNA. (A) Western blot analysis of FOSB protein expression levels. The three different FOSB siRNA transfectants (siRNA1, siRNA2 and siRNA3) and the empty vector transfectant (vector) are shown. (B) Cell viability was evaluated with MTT assay using absorbance readings at $490 \mathrm{~nm}$. SGC7901 cells were transfected with FOSB siRNA or empty vector for 1, 2, 3 and 4 days, respectively. The values are indicated by the mean of three determinations. (C) Colony numbers of the SGC7901/siRNA and control cells in plate colony formation assays. The data represent the means \pm SEM of three independent experiments. (D) Transwell assays demonstrated that silencing of FOSB expression by siRNA promoted cell migration in SGC7901 cells ("P<0.05). Representative photos of stained cells are shown with the original magnification of $\mathrm{x} 100$.

Table II. Univariate and multivariate analyses of prognostic factors in gastric cancer.

\begin{tabular}{|c|c|c|c|c|c|c|}
\hline \multirow[b]{2}{*}{ Variables } & \multicolumn{3}{|c|}{ Univariate analysis } & \multicolumn{3}{|c|}{ Multivariate analysis } \\
\hline & $\mathrm{RR}$ & $95 \% \mathrm{CI}$ & P-value & RR & $95 \% \mathrm{CI}$ & P-value \\
\hline Gender (Male vs. female) & 1.428 & $1.049-1.827$ & 0.126 & - & - & - \\
\hline Age ( $<60$ vs. $\geq 60$ years $)$ & 1.336 & $1.023-2.062$ & 0.057 & - & - & - \\
\hline Tumor size $(<5 \mathrm{vs} . \geq 5 \mathrm{~cm})$ & 1.137 & $0.810-1.618$ & 0.164 & - & - & - \\
\hline Location (Cardia vs. body/antrum) & 1.523 & $1.184-1.843$ & 0.504 & - & - & - \\
\hline Histological grade (Poor vs. well + mod) & 0.675 & $0.426-0.944$ & $0.022^{\mathrm{a}}$ & 0.527 & $0.376-0.926$ & 0.807 \\
\hline Distant metastasis (+ vs. -) & 1.879 & $1.079-2.682$ & 0.326 & - & - & - \\
\hline Invasive depth (T3+ T4 vs. T1 + T2) & 1.077 & $0.864-1.563$ & $<0.001^{\mathrm{a}}$ & 1.656 & $1.023-2.543$ & 0.956 \\
\hline TNM stage (III + IV vs. I + II) & 1.758 & $0.984-2.197$ & $0.032^{\mathrm{a}}$ & 0.649 & $0.284-0.914$ & $0.016^{\mathrm{a}}$ \\
\hline Lymph node metastasis (+ vs. -) & 1.233 & $0.824-1.860$ & $0.004^{\mathrm{a}}$ & 0.418 & $0.240-1.048$ & 0.608 \\
\hline FOSB expression (Low vs. high) & 0.332 & $0.204-0.605$ & $<0.001^{\mathrm{a}}$ & 0.430 & $0.324-0.695$ & $<0.001^{\mathrm{a}}$ \\
\hline
\end{tabular}

HR, hazard ratio; CI, confidence interval; Well, well-differentiated; Mod, moderately differentiated; Poor, poorly differentiated. ${ }^{\text {a }} \mathrm{P}-\mathrm{value}<0.05$ was considered statistically significant.

SGC7901 cells compared with the control cells (Fig. 5B and $\mathrm{C} ; \mathrm{P}<0.05)$. In addition, knockdown of FOSB expression dramatically reduced the migrated cell number of SGC7901 cells by Transwell assay (Fig. 5D; $\mathrm{P}<0.05$ ).
FOSB expression is correlated to clinicopathological factors in $G C$. Chi-square test was used to analyze the correlation between the FOSB expression in GC tissues and various clinicopathological characteristics. Table I, indicates that we found 


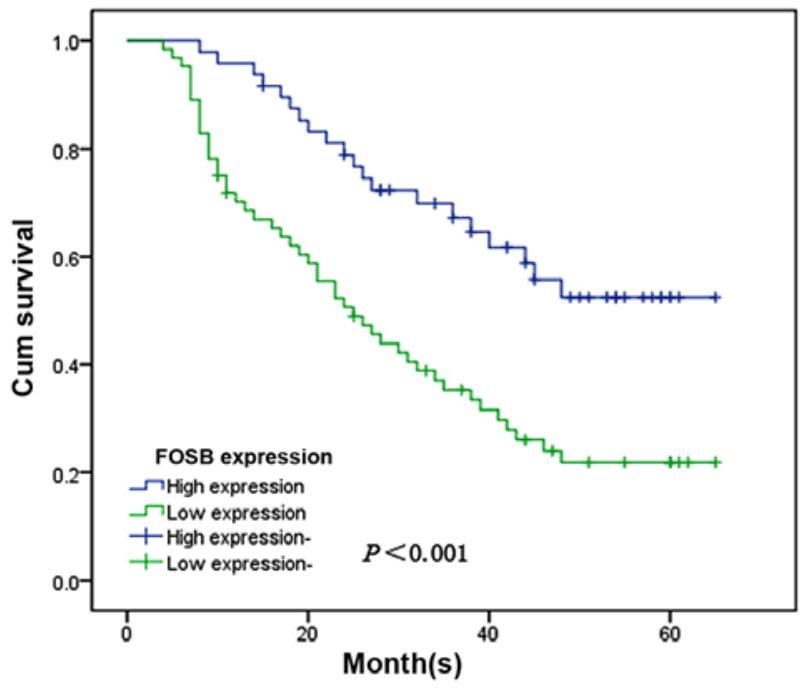

Figure 6. Kaplan-Meier survival curves according to FOSB level. Patients with low FOSB expression had a significantly poorer prognosis than those with high FOSB expression $(\mathrm{P}<0.001, \log$-rank test $)$.

that decreased expression of FOSB was significantly correlated to poor differentiation $(\mathrm{P}<0.001)$, lymph node metastasis $(\mathrm{P}=0.001)$ and high TNM stage $(\mathrm{P}=0.021)$. However, there was no statistically significant correlation between FOSB expression and gender, age, tumor size, location, invasive depth, $H$. pylori infection and distant metastasis $(\mathrm{P}>0.05)$. To further estimate the correlation between the FOSB expression and the prognosis of GC patients, we used Kaplan-Meier survival curves and the log-rank test to investigate the prognostic effect of FOSB on the overall survival rate of GC patients by comparing the 5 -year survival rate of patients with high or low levels of FOSB expression. The patients with the low levels of FOSB expression had a poorer prognosis than the patients with high levels of FOSB expression $\left(\chi^{2}, 13.421\right.$, $\mathrm{P}<0.001$; Fig. 6).

Univariate and multivariate analysis was performed to evaluate the independent prognostic roles of FOSB (Table II). Univariate Cox regression analysis showed that histological grade, invasive depth, TNM stage, lymph node metastasis and FOSB expression were significantly associated with overall survival in GC patients. Moreover, multivariate Cox regression analysis confirmed FOSB expression and TNM stage as independent predictors of the overall survival of GC patients.

\section{Discussion}

Gastric cancer has one of the highest incidence rates in the world, and the 5-year survival rate of is less than $20-25 \%$ in China because of frequent relapse and high metastasis rate postoperatively (16), and most patients present advanced disease at diagnosis making its treatment very intricate (17). It is widely accepted that early diagnosis and treatment are keys for better clinical outcome in patients with gastric cancer (18). There have been many advances in diagnostics and therapeutics in GC, however, dismal prognosis, despite these improvements, still persist (19). Many cancer-related molecules have been characterized with the goal of developing novel anticancer therapies, including targeted drugs or antibodies and cancer vaccines (20). FosB is known as a kind of the biological markers, but there is no related report on FOSB in GC.

FOSB is a member of the Fos gene family. It is activated by growth factors and is a nuclear protein of 338-amino acids that shows $70 \%$ homology with Fos. Furthermore, like Fos, it forms complexes with the Jun-family of transcription factors 8 , suggesting that it is involved in regulating gene expression (6). Together with the Jun family members, the Fos family of transcription factors form the group of AP-1 proteins which, after dimerization, bind to so-called TPA-responsive elements in the promoter and enhancer regions of target genes (21). In contrast to Jun proteins, Fos family members are not able to form homodimers, but heterodimerize with Jun partners, giving rise to various trans-activating or trans-repressing complexes with different biochemical properties (22). AP-1 in turn controls a number of cellular processes including differentiation, proliferation and apoptosis (7). To further analyze the role of the FOSB transcription factor in GC and to explore whether the level of FOSB expression is involved in GC cell viability, proliferation and migration, we used immunohistochemistry on paraffin sections of 116 gastric carcinomas. According to our new measurements, we demonstrated that FOSB was expressed higher in adjacent non-cancerous tissues than in GC tissues. In addition, we also found that a downregulated expression of FOSB was markedly connected with advanced TNM stage, poor differentiation and lymph node metastasis. Thus, the abnormal expression of FOSB might be involved in GC tumor progression and metastasis, and we speculated that FOSB may play a tumor suppressor role in GC. Subsequently, qPCR and western blot analysis were used to find that FOSB expression was decreased at the mRNA and protein levels in most cancer tissues compared to their adjacent non-cancerous tissues. Moreover, we showed that the viability, proliferation and migration in vitro of MKN45 cells were observably inhibited by overexpression of FOSB, whereas knockdown of FOSB expression promoted the viability, proliferation and migration of SGC7901 cells in vitro. Furthermore, it is well known that a high prevalence of $H$. pylori is always accompanied by a high incidence of gastric cancer (23). One study demonstrates that $H$. pylori positivity is a beneficial prognostic indicator in patients with gastric cancer (24). However, in the present study, there was no significant discrepancy in the expression of FOSB in the patients with and without $H$. pylori infection.

In accordance with the Kaplan-Meier survival, it showed that low FOSB expression had significant relationship with shorter survival time of GC patients. Multivariate analysis suggested that in patient overall survival, FOSB expression was an independent prognostic indicator. Moreover, in a study on human keratinocytes, the calcium-induced differentiation and expression of the differentiation marker involucrin was accompanied by an increase of FOSB and a decrease of Fra-1 expression (13). Regarding the functional differences between Fos family members, FOSB is the only AP-1 protein which cannot bind and inactivate glucocorticoid receptors (25). Vice versa, unlike other AP-1 family members, FOSB proteins cannot be blocked by GR proteins which are expressed in the epithelial cells of terminal lobular units and which play a role in the differentiation of mammary epithelial cells $(26,27)$. 
The knowledge on FOSB is still not complete, thus, further research on the effect of FOSB will be necessary. The mechanisms which lead to the downregulated expression of FOSB in GC patients still require further investigation.

The above data show that the downregulated expression of FOSB is involved in tumor progression of GC patients and might represent a prognostic indicator. Thus, extra experiments will be necessary to prove this hypothesis and that FOSB may play a useful role in new therapeutic interventions in $\mathrm{GC}$ patients.

\section{References}

1. Ajani JA, Bentrem DJ, Besh S, D'Amico TA, Das P, Denlinger C, Fakih MG, Fuchs CS, Gerdes H, Glasgow RE, et al; National Comprehensive Cancer Network: Gastric cancer, version 2.2013: Featured updates to the NCCN Guidelines. J Natl Compr Canc Netw 11: 531-546, 2013.

2. Lin Y, Ueda J, Kikuchi S, Totsuka Y, Wei WQ, Qiao YL and Inoue M: Comparative epidemiology of gastric cancer between Japan and China. World J Gastroenterol 17: 4421-4428, 2011.

3. Howlett M, Menheniott TR, Judd LM and Giraud AS: Cytokine signalling via gp130 in gastric cancer. Biochim Biophys Acta 1793: 1623-1633, 2009.

4. Siderovski DP, Blum S, Forsdyke RE and Forsdyke DR: A set of human putative lymphocyte $\mathrm{G}_{0} / \mathrm{G}_{1}$ switch genes includes genes homologous to rodent cytokine and zinc finger protein-encoding genes. DNA Cell Biol 9: 579-587, 1990.

5. Martin-Gallardo A,McCombie WR, Gocayne JD,FitzGeraldMG, Wallace S, Lee BM, Lamerdin J, Trapp S, Kelley JM, Liu LI, et al: Automated DNA sequencing and analysis of 106 kilobases from human chromosome 19q13.3. Nat Genet 1: 34-39, 1992.

6. Zerial M, Toschi L, Ryseck RP, Schuermann M, Müller R and Bravo R: The product of a novel growth factor activated gene, fos $\mathrm{B}$, interacts with JUN proteins enhancing their DNA binding activity. EMBO J 8: 805-813, 1989.

7. Hess J, Angel P and Schorpp-Kistner M: AP-1 subunits: Quarrel and harmony among siblings. J Cell Sci 117: 5965-5973, 2004.

8. Krosl J and Sauvageau G: AP-1 complex is effector of Hox-induced cellular proliferation and transformation. Oncogene 19: 5134-5141, 2000

9. Mechta F, Lallemand D, Pfarr CM and Yaniv M: Transformation by ras modifies AP1 composition and activity. Oncogene 14: 837-847, 1997.

10. Pospelova TV, Medvedev AV, Kukushkin AN, Svetlikova SB van der Eb AJ, Dorsman JC and Pospelov VA: E1A + cHa-ras transformed rat embryo fibroblast cells are characterized by high and constitutive DNA binding activities of AP-1 dimers with significantly altered composition. Gene Expr 8: 19-32, 1999.

11. Chung JY, Huang C, Meng X, Dong Z and Yang CS: Inhibition of activator protein 1 activity and cell growth by purified green tea and black tea polyphenols in H-ras-transformed cells: Structureactivity relationship and mechanisms involved. Cancer Res 59: 4610-4617, 1999.
12. Darne C, Martinez A, Lallemand D, Morel L, Jean C, Saru JP, Schmid HP and Manin M: Down-regulation of AP1 activities after polarization of vas deferens epithelial cells correlates with androgen-induced gene expression. J Steroid Biochem Mol Biol 72: 103-113, 2000.

13. Ng DC, Shafaee S, Lee D and Bikle DD: Requirement of an AP-1 site in the calcium response region of the involucrin promoter. $\mathrm{J}$ Biol Chem 275: 24080-24088, 2000.

14. Milde-Langosch K, Kappes H, Riethdorf S, Löning T and Bamberger AM: FosB is highly expressed in normal mammary epithelia, but down-regulated in poorly differentiated breast carcinomas. Breast Cancer Res Treat 77: 265-275, 2003.

15. Kim JH, Lee JY, Lee KT, Lee JK, Lee KH, Jang KT, Heo JS, Choi SH and Rhee JC: RGS16 and FosB underexpressed in pancreatic cancer with lymph node metastasis promote tumor progression. Tumour Biol 31: 541-548, 2010.

16. Wang J, Yu JC, Kang WM and Ma ZQ: Treatment strategy for early gastric cancer. Surg Oncol 21: 119-123, 2012.

17. Mahar AL, Coburn NG, Singh S, Law C and Helyer LK: A systematic review of surgery for non-curative gastric cancer. Gastric Cancer 15 (Suppl 1): S125-S137, 2012.

18. Piazuelo MB and Correa P: Gastric cáncer: Overview. Colomb Med (Cali) 44: 192-201, 2013.

19. Jemal A, Bray F, Center MM, Ferlay J, Ward E and Forman D: Global cancer statistics. CA Cancer J Clin 61: 69-90, 2011.

20. van Seventer GA, Salmen HJ, Law SF, O'Neill GM, Mullen MM, Franz AM, Kanner SB, Golemis EA and van Seventer JM: Focal adhesion kinase regulates betal integrin-dependent $\mathrm{T}$ cell migration through an HEF1 effector pathway. Eur J Immunol 31: 1417-1427, 2001.

21. Milde-Langosch K: The Fos family of transcription factors and their role in tumourigenesis. Eur J Cancer 41: 2449-2461, 2005.

22. Ryseck RP and Bravo R: c-JUN, JUN B, and JUN D differ in their binding affinities to AP-1 and CRE consensus sequences: Effect of FOS proteins. Oncogene 6: 533-542, 1991.

23. Yamaoka $Y$, Kato $M$ and Asaka M: Geographic differences in gastric cancer incidence can be explained by differences between Helicobacter pylori strains. Intern Med 47: 1077-1083, 2008.

24. Wang F, Sun GP, Zou YF, Zhong F, Ma T, Li XQ and Wu D: Helicobacter pylori infection predicts favorable outcome in patients with gastric cancer. Curr Oncol 20: e388-e395, 2013.

25. Lucibello FC, Slater EP, Jooss KU, Beato M and Müller R: Mutual transrepression of Fos and the glucocorticoid receptor: Involvement of a functional domain in Fos which is absent in FosB. EMBO J 9: 2827-2834, 1990.

26. Angel P and Karin M: The role of Jun, Fos and the AP-1 complex in cell-proliferation and transformation. Biochim Biophys Acta 1072: 129-157, 1991.

27. Rosen JM, Zahnow C, Kazansky A and Raught B: Composite response elements mediate hormonal and developmental regulation of milk protein gene expression. Biochem Soc Symp 63: 101-113, 1998. 